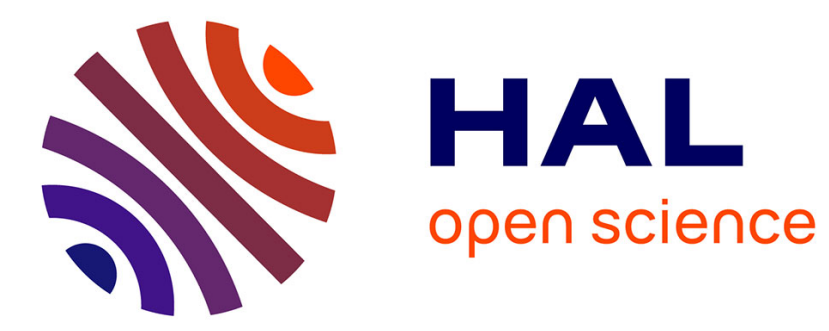

\title{
Petite fabrique géo-artistique des espaces publics et des territoires
}

\author{
Luc Gwiazdzinski
}

\section{To cite this version:}

Luc Gwiazdzinski. Petite fabrique géo-artistique des espaces publics et des territoires. L'Observatoire, la revue des politiques culturelles , 2016, 48, pp.32-38. 10.3917/lobs.048.0032 . hal-01523010

\section{HAL Id: hal-01523010 https://hal.science/hal-01523010}

Submitted on 15 May 2017

HAL is a multi-disciplinary open access archive for the deposit and dissemination of scientific research documents, whether they are published or not. The documents may come from teaching and research institutions in France or abroad, or from public or private research centers.
L'archive ouverte pluridisciplinaire HAL, est destinée au dépôt et à la diffusion de documents scientifiques de niveau recherche, publiés ou non, émanant des établissements d'enseignement et de recherche français ou étrangers, des laboratoires publics ou privés. 


\section{Petite fabrique géo-artistique des espaces publics et des territoires}

L’Observatoire n²48, été 2016, pp. 32-38

\section{Luc Gwiazdzinski (*)}

« La valeur des villes se mesure au nombre des lieux qu'elles réservent à l'improvisation ${ }^{1}$. »

Le philosophe Jean-Paul Dollé nous a prévenus : « La géographie n’est pas une connaissance facile [...]. Il faut fendre les mots du monde, oser aller voir ailleurs. » Dans le contexte mouvant entre « art à l'état gazeux ${ }^{2}$ » et métropoles « liquides ${ }^{3}$ » de nouveaux acteurs, de nouvelles pratiques hybrides associant art et ville, création artistique et production urbaine émergent. De nouveaux collectifs pluridisciplinaires s'organisent, d'autres protocoles se déploient, d'autres agencements et territorialités éphémères et multiscalaires s'inventent qui restent encore largement impensées.

Notre réflexion démarre au tournant du XXIe siècle à un moment charnière pour les arts, la fabrique de la ville et des territoires, les modes de vie et attentes des citoyens. Le champ artistique et culturel, dimension importante des activités humaines et domaine d'actions publiques ou privées, est marqué par des transformations majeures. Un « art contextuel », en rupture avec l'art classique, se déploie. Les institutions culturelles investissent dans le « hors les murs ». Nombre d'artistes sortent de leurs ateliers pour s'exprimer à l'extérieur et les arts de la rue se muent peu à peu en arts urbains. Côté fabrique de la métropole et recherche urbaine, urbanistes, aménageurs et géographes sont en quête de nouvelles clés de lecture de « l'outre-ville $»^{5}$. Ils portent une attention nouvelle aux enjeux sociaux, à la cohésion sociale, aux temporalités, au milieu, à l'habiter et au rapport anthropologique à l'espace, aux usages, au quotidien urbain et à la participation des habitants.

Prenant conscience de l'importance de l'art et de la culture comme leviers économiques, activateurs de lien social et outils d'urbanisme et d'aménagement des territoires, les collectivités et pouvoirs publics ont peu à peu cherché à accompagner cette effervescence et, plus récemment, tenté de la documenter ${ }^{6}$ pour réorienter leurs politiques ou « outiller » les acteurs locaux. Enfin, on constate une forte demande pour l'art et les artistes dans de nombreux domaines, et un besoin de rencontre dans les métropoles post-modernes où les grands rythmes collectifs qui scandaient la vie sociale se sont atténués.

Au croisement de ces attentes et de ces mouvements, sont apparus des tiers acteurs d'un nouveau genre qui ont le territoire et l'espace public comme scènes et les protocoles géographiques comme points communs : les géo-artistes ${ }^{7}$. Avec eux, dans l'entre-deux et hors les murs, l'espace public devient à la fois le lieu de croisements entre les acteurs de la fabrique urbaine, une scène artistique et l'objet de métamorphoses néo-situationnistes. 


\section{Galaxie géo-artistique}

Ces nouveaux praticiens urbains déploient leurs savoir-faire hors les murs à travers la France, l'Europe et le monde comme s'il était plus facile de collaborer sur le terrain que dans les ateliers ou dans les agences. Ces géo-artistes aux formations variées (designers, scénographes, artistes de rue, danseurs-chorégraphes, artistes lumière, plasticiens contextuels, architectes, urbanistes, géographes, etc.) qui s'aventurent dans l'espace public forment une nouvelle galaxie aux limites floues.

Ils œuvrent le plus souvent dans le cadre de collectifs hybrides, adaptables aux situations et en évolution constante, loin du mythe de l'artiste qui fait et décide seul. Leurs noms mêmes sont un manifeste pour d'autres relations expérimentales art-espace et art-urbanisme : Ici-Même Grenoble, Ici-Même Paris, Cie Off, Bruit du frigo, YA+K, Échelle inconnue, Exyst, KompleX KapharnaüM, Laboratoire, La Folie kilomètre, Asphalt Piloten, Bellastock, Agence Nationale de psychanalyse urbaine, Ilotopie, Yes We Camp, Collectif Etc, AWP, Bazar urbain, Agence touriste, , Tabula Rasa, Opéra Pagaï ou Bivouac urbain. Ces collectifs pluridisciplinaires sont à l'image de KompleX KapharnaüM : «Équipe de plasticiens, vidéastes, écrivains et musiciens », de Yes We Camp " collectif ouvert, en évolution constante, mêlant architectes, plombiers, urbanistes, charpentiers, ingénieurs, administrateurs, artistes, jardiniers, bricoleurs, régisseurs de spectacle, graphistes, etc. " ou d'Exyst, créé en 2003 par des architectes, qui regroupe aujourd'hui des graphistes, vidéastes, photographes, Dj's, botanistes et constructeurs. Dans cette galaxie, on trouve des danseurs et chorégraphes comme Annick Charlot de la Cie Acte, Philippe Saire, Yann Lheureux à Montpellier ou la regrettée Odile Duboc à Belfort. Il s'agit parfois d'individus comme Nicolas Simarik (auteur notamment du Catalogue de la déroute), Stefan Shankland (inventeur du concept de «HQAC »), Olivier Darné et ses abeilles, les artistes lumière Yann Kersalé ou Gianni Ravelli, le marcheur Henrik Sturm ou Joël Henry du Laboratoire de tourisme expérimental. Tous œuvrent avec des équipes qui se coalisent en fonction des projets, en lien avec des organisateurs d'événements et de manifestations avec lesquels ils composent.

Leurs interventions expérimentales sous forme de spectacles, d'architectures éphémères, d'installations ou d'interventions plastiques, s'inscrivent dans les champs de la culture, de l'animation mais également dans celui de l'urbanisme. Leurs commanditaires sont majoritairement des collectivités, parfois l'État, des institutions publiques et parapubliques, des entreprises de la construction ou du transport et des fondations dans le cadre de projets artistiques, voire d'appels à projets associant la dimension artistique. Leurs interventions artistiques s'intègrent parfois à des dispositifs de long terme comme les ZAT (Zones artistiques temporaires) à Montpellier, des résidences avec le Pôle des arts urbains (pOlau), Lieux publics à Marseille ou Itinéraires bis dans les Côtes d'Armor, des festivals comme ceux de Chalon ou d'Aurillac, des opérations culturelles comme Marseille Provence 2013 ou dans la programmation hors les murs de scènes nationales comme l'Hexagone ou le Merlan.

Les pratiques, les modes d'intervention et l'esthétique développés se rapprochent parfois d'autres organisations comme la 27e Région " laboratoire de transformation publique des Régions de France » qui œuvre dans le design des politiques publiques, d'associations d'éducation populaire comme Robins des villes, d'agences comme Dédale qui s'intéresse à 
l'innovation, voire du Pôle d'exploration des ressources urbaines (PEROU), « laboratoire de recherche-action sur la ville hostile » et des approches de l'architecte Patrick Bouchain. À travers une esthétique de la bricole et du temporaire, les démarches géo-artistiques sont également en résonance avec d'autres occupations temporaires des espaces publics depuis les tentes des Enfants de Don Quichotte sur le canal Saint- Martin en 2006 jusqu'à la Révolution des parapluies de Hong Kong en passant par les places de New York, de Grèce, de Turquie ou d'Ukraine, les Printemps arabes et Nuit debout ${ }^{8}$, voire avec certaines pratiques sportives (parkour, roller, skate, etc.) qui investissent l'espace urbain, transforment et augmentent son usage. Leurs protocoles rejoignent la mode de l'expérience corporelle et de l'immersion en journalisme ou en littérature. C'est comme si l'éprouver, la rencontre et l'intervention se paraient d'une dimension réelle, celle du « territoire profond » et des «vrais gens ». Au même moment, la géographie retrouve le terrain et le « plein vent » et explore les « territoires apprenants ${ }^{9} »$.

\section{Caractères communs}

Ces collectifs pluriels ont un certain nombre de points communs : intervention dans l'espace public, action collective et collaborative, interdisciplinarité et démarches in situ qui répondent aux questions de développement durable, participent à la définition de nouveaux rapports entre art et société, à une nouvelle esthétique, à un besoin d'être ensemble, voire de partager une utopie concrète sur la ville et le vivre ensemble.

Ces nouvelles pratiques et ces nouveaux praticiens ont l'espace public politique et architectural en commun, terrain de jeu et d'expérimentation. Ils investissent le territoire en tant que matériau et atelier. Ils ont également le « faire » en commun, en lien avec la culture Do it Yourself et l'économie du partage. Ils mettent en avant le malléable, l'itinérant et le bricolage, cette forme d'innovation ordinaire, d'ingéniosité quotidienne qui laisse une place à la figure de l'amateur, au bricoleur, celui qui s'arrange avec les « moyens du bord », c'est-à-dire un ensemble " à chaque instant fini d'outils et de matériaux, hétéroclites au surplus ${ }^{10}$ ». Les mécanismes de coproduction sont souvent valorisés comme l'« expertise quotidienne » des participants, détenteurs de savoirs et de compétences distincts de l'expertise des élites et le " vernaculaire " ", c'est-à- dire tout ce qui tend à agencer de manière optimale, les ressources et les matériaux disponibles pour construire ou aménager dans une logique qui rejoint le circuit court.

L'expérimentation et la participation sont centrales. Le Collectif Etc qui s'est fait notamment connaître par son Détour de France ${ }^{12}$, se dit « support d'expérimentations urbaines participatives ». Les paysagistes de Coloco sont " explorateurs de la diversité urbaine à partir d'architectures, paysages, films et installations ». Les performeurs et plasticiens d'Agence Touriste, proposent d'autres manières de lire et de réinventer la ville. Ces démarches font souvent appel à une expertise citoyenne et habitante en amont. Elles se veulent ascendantes et participatives, sollicitent les usagers - habitants et passants - invités à co-construire avec les artistes. Elles prennent la forme de performances mais aussi de happenings en interaction avec le public. Les géo-artistes développent un art qui se donne à voir et un art qui s'expérimente et se vit comme celui du mouvement Fluxus.

Ils s'appuient sur des protocoles, des dispositifs, des événements, des interventions artistiques et botaniques, créant des décalages qui perturbent ou incitent à changer de regard ou 
d'usage. Ils créent des mobiliers urbains, des scénographies, des mises en scène in situ et des parcours qui leur permettent d'entrer dans la vie simple des gens et dans le temps local et contribuent à la fabrique d'une autre cité, à l'émergence d'une « ville métaphorique ${ }^{13}$ ». Héritiers de Georges Perec, Julio Cortázar, François Maspero, Pierre Sansot, Jean-François Augoyard et du collectif Stalker, ils sont imités par les urbanistes qui se réclament du même héritage, s'emparent des parcours urbains et les déclinent désormais en amont de tous leurs projets.

Ces interventions qui s'inscrivent généralement dans le quotidien urbain ${ }^{14}$, l'existence ordinaire et collective exhalent une certaine poésie de l'instant. Ce sont des moments ludiques, une récréation, un jeu où « le sujet joue à croire, à se faire croire ou à faire croire aux autres qu'il est un autre que lui-même ${ }^{15}$ » et parfois une fête, cette promesse de l'exception chère à Georges Bataille.

Elles s'invitent dans la complexité métropolitaine, au centre comme sur les marges, en journée comme la nuit, investissant les centres des villes, les entre-temps, les no man's land et autres no man's time comme les chantiers. Ces artistes déploient des stratégies et des tactiques spatiales et se font géographes. Ils tracent des parcours et fabriquent des représentations cartographiques qui mobilisent d'autres imaginaires comme dans les ateliers " nappemondes 》 du collectif Ici-Même Grenoble, ou dans les travaux de l'artiste marcheur Mathias Poisson avec ses cartes subjectives.

Ces interventions jouent souvent sur le décalage, l'émotion et les sens, dimensions longtemps négligées en urbanisme et architecture. L'œuvre devient participative et permet une pluralité de l'expérience au sens de "faire l'essai de ». Elles sont multisensorielles ou nous privent d'un sens, comme dans les parcours d'exploration de la ville les yeux bandés. Elles invitent à la dégustation du territoire avec le " miel béton » d'Olivier Darné. L'événement est parfois parade et mouvement, musiques, lumières, senteurs et saveurs. Certaines expériences construisent une esthétique foraine et circassienne du mouvement, une fête des sens pour un spectateur consentant qui se laisse embarquer.

Ces interventions sont généralement modestes et temporaires. Elles deviennent parfois monumentales comme avec les machines de Royal de Luxe ou les mises en scène d'Illotopie et peuvent mobiliser une foule importante lors d'événements culturels internationaux exceptionnels. Elles ne sont pas toutes transgressives sauf peut-être quand elles organisent la présence de l'intime dans l'espace public comme pour l'expérience Collection de collections, de Laboratoire.

Ce faisant, les praticiens se posent en néo-situationnistes, designers de « situations », ce « moment de la vie, concrètement et délibérément construit par l'organisation collective d'une ambiance unitaire et d'un jeu d'événements ${ }^{16}$. Ils sont des « ambianceurs » qui mobilisent l'émotion, des créateurs qui inventent, des poètes qui décalent et des forains- bonimenteurs, à la fois enchanteurs et arnaqueurs de passants consentants.

\section{Apports pour les habitants et la fabrique de la ville}

Ces géo-artistes ne sont pas de simples décorateurs. Leurs interventions dépassent l'action cosmétique ou événementielle dans laquelle pourrait les enfermer certains commanditaires. Leur 
impact ne se limite pas aux interventions sur de nouveaux quartiers ou des friches, ou dans la mise en scène d'une manifestation publique ni au moment de l'événement mais se décline en amont et en aval.

Les géo-artistes braconnent, repèrent, décryptent, désignent, produisent, mobilisent, mettent en désir, rassemblent, lient, jouent, font jouer un public le plus souvent non captif et hétérogène. Avec eux, la même ville devient une autre. L'ailleurs s'invite ici pour un instant. Leurs protocoles d'exploration permettent de découvrir un territoire dans la complexité de ses espaces et de ses temporalités. Les mises en scène permettent au public de se décaler et de voyager sur place, explorant un « exotisme de proximité ». L'artiste nous invite à changer de regard sur nos villes et sur nos vies quotidiennes et nous pousse à sortir des trajets quotidiens ou des figures touristiques imposées pour retrouver le goût de la ville et des autres.

Qu'il investisse une rue ou qu'il parcourt la ville, l'événement enchante le quotidien, transfigure le réel et humanise l'espace public. Pour quelques heures ou quelques jours, les mises en scène métamorphosent tout ou partie d'un quartier ou d'une rue. Les géo-artistes participent à la transformation (sociale, symbolique, matérielle etc.) des espaces, ce processus «plus riche que l'idée de révolution, qui en garde la radicalité transformatrice, mais la lie à la conservation ${ }^{17}$ » et à leur ludification. Les dispositifs qu'ils déploient améliorent temporairement le confort, l'ergonomie et l'hospitalité des espaces publics et peuvent ensuite être déclinés de manière pérenne.

Leurs interventions artistiques sont le plus souvent la source d'une véritable expérience. L'événement qui développe des « interactions " ${ }^{18}$, tisse des liens où il n'y en avait pas, crée des collectifs là où régnait l'anonymat. Ils créent des "spatialités artistiques temporaires », des « artétopes 19» à des moments et sur des espaces sans intérêt. Souvent proches du concept de spectacle vivant, ce « partage du sensible » ${ }^{20}$, permet de former des « communautés d'expérience ${ }^{21}$ et de développer un sentiment d'appartenance en « faisant consistance » par les affects ${ }^{22}$.

En imaginant des situations contre lesquelles " on se cogne », les géo-artistes nous permettent de nous réapproprier le réel, « ce que l'on n'attendait pas ${ }^{23}$ ». Par l'événement, ils suscitent notre attention. Ces expériences font événement, comme irruption de l'intermittence, aussi bien dans le cours de la vie de l'individu que d'une communauté d'individu. Hors là, hors les murs, hors sol, hors normes, les géo-artistes « nous invitent à être », à habiter, à exister, c'està-dire à « avoir sa tenue hors de soi, dans l'ouverture ${ }^{24}$ ».

À leur manière, ces artistes fabriquent les lieux d'une «créativité dispersée » et d'une expérience esthétique. Leurs interventions dépassent les « terminaux de consommation culturelle » qui ne s'intéressent qu'à la rencontre entre les œuvres/produits et le public/consommateur et les « nouveaux territoires de l'art », friches, fabriques, espaces intermédiaires ou squats qui avaient déjà placé l'expérimentation artistique au cœur des expérimentations sociale, économique, urbaine et politique. Au-delà de la métamorphose temporaire, ils participent plus largement à la lecture et à l'écriture des métropoles contemporaines.

Ils recomposent la géographie complexe des espaces métropolitains sur lesquels ils interviennent : centres-villes ripolinés, zones prioritaires de la politique de la ville, friches industrielles, interstices, cours d'eau, forêts, zones agricoles et restes de « nature » rattrapés par 
l'urbanisation. Ils s'associent à la redéfinition des polarités urbaines et à la production d'espaces publics d'intérêt métropolitain. Ils dessinent la ville au-delà de la ville, facilitent son « imagibilité ${ }^{25}$ et son appropriation (comme dans le cas de l'intervention d'Ilotopie en Camargue) dans ses espaces et dans ses temps.

Leurs performances, les événements qu'ils mettent en scène et « designent » viennent alors sculpter les temps de la ville. Ils dessinent l'agenda d'une « métropole intermittente ${ }^{26} »$ avec ses spatialités temporaires éclatées dans le temps et dans l'espace. Ces nouvelles formes et agencements temporaires permettent un autre déploiement de l'être ensemble et du collectif. Ils font collectif et territoire dans une société où tout paraît mobile, fluctuant et affaire individuelle, et ce, dans un présent axé sur le culte de l'éphémère et des projets à court terme. La multiplication d'événements permet à tout ou partie de la ville de maintenir une illusion de lien social et le sentiment de vivre ensemble.

Les géo-artistes s'engagent dans l'animation de la ville « lieu de maximisation des interactions ${ }^{27}$ » en injectant de la surprise, du détournement, de la ruse et humanisent l'espace public. Par leurs expérimentations, ils incitent les autorités et les urbanistes à imaginer un urbanisme plus sensible et à co-construire avec les usagers un mobilier urbain et des espaces publics modulaires et polyvalents en s'appuyant sur quelques grands principes comme l'hospitalité, l'information, la qualité, la sensibilité, la variété, l'inattendu, l'alternance, la sécurité par l'accroissement de la présence humaine et l'enchantement par l'invention permanente.

\section{Nouvelles géographies, nouveaux récits}

La décentralisation et la séparation progressive entre la culture et l'État, l'émergence de territoires et de métropoles qui travaillent en réseau, l'efface- ment des grands récits nationaux créent une demande de récits locaux fédérateurs notamment à l'échelle des métropoles ${ }^{28}$. À travers leurs interventions expérientielles et émotionnelles, les géo-artistes développent des mises en récit, des fictionnalisations territoriales et un imaginaire susceptibles de faire bouger les lignes. Au-delà du récit d'urbanisme, ils sont également partie prenante dans l'émergence d'un " urbanisme fictionnel $»^{29}$, qui tend à substituer une production narrative à une production réelle de ville et de territoire.

Ces interventions laissent des traces dans l'espace public politique, dans l'espace public physique des urbanistes et dans l'espace public de la toile dont l'archéologie reste à faire. Elles inspirent de nouvelles politiques publiques : Paris Plages, musées itinérants, Université foraine, etc. Parfois, ces expériences artistiques finissent par se matérialiser de manière plus pérenne comme à Marseille avec le sentier de randonnée métropolitain et à Nantes où les interventions de Royal de Luxe ont fini par accoucher d'un quartier. À Paris, la fermeture dominicale des Champs-Élysées aux voitures, l'allongement des horaires de métro en soirée et toutes les nouvelles utilisations malléables de la ville doivent beaucoup à ces expérimentations.

Le géo-artiste déplace les centralités, s'emploie à recréer l'espace public et participe à l'émergence d'une nouvelle ergonomie de la "ville à la carte ". Ses interventions in vivo contredisent la banalisation et l'uniformisation des espaces urbains. En sortant des galeries d'art pour s'approprier tous les espaces des centres et des marges, il se positionne, prend date et prend 
position sur la ville. Il oblige tout le monde à bouger et fait évoluer la réflexion urbaine jusque dans les laboratoires de recherche. En pédagogie, on peut militer avec eux pour que l'appropriation de la ville à l'école se fasse par la pratique, les « classes de ville » et le jeu urbain.

Ces « marginaux sécants ${ }^{30} »$, partie prenante dans plusieurs systèmes d'action, jouent naturellement le rôle d'intermédiaires et d'interprètes entre des acteurs, en mettant du jeu et du plaisir là où il n'y a souvent que technique et froideur. La dimension participative et expérientielle in vivo permet un renouvellement du dialogue élu/artiste. Leur statut hybride oblige à enrichir notre vocabulaire, à détourner des mots d'un champ vers l'autre (communautés d'expérience, chantier, atelier, immersion, résidence, etc.) ou à les associer pour mieux ruser : Géo-choré-graphie ; Géo-artistes, Haute qualité Artistique et culturelle (HQAC) ; Résidence de géographe ou de Praticiens urbains peu ordinaires.

Dans un contexte de concurrence territoriale exacerbée, leurs interventions dans différentes manifestations et festivals, participent à la « ville événementielle » et aux stratégies de marketing urbain. Elles sont utilisées comme outils légers dans les processus de revalorisation des centres urbains, de marketing et de renforcement de l'attractivité économique et touristique à côté d'autres approches comme la patrimonialisation ou «l'architecture iconique ${ }^{31}$ ». Localement, elles sont également mobilisées pour renforcer le sentiment d'appartenance, la fierté territoriale et pour modifier l'image de tout ou partie d'une ville.

\section{Dures limites}

Ces convergences et hybridations dans l'espace public participent à la fois d'une " géographie pragmatique » et des " arts du territoire ", deux champs d'inconfort et de mise à l'épreuve. Il faut naturellement s'interroger sur les risques et les limites de ces démarches par rapport à un discours normatif sur la « ville créative».

Connaissant la capacité de récupération des thèmes de la critique artiste ${ }^{32}$ par le système capitaliste, on peut facilement repérer les risques d'instrumentalisation par les politiques, les politiques publiques, le secteur touristique ou l'immobilier. Les géo-artistes s'inscrivent dans des logiques de marketing territorial qui les dépassent.

Ils pointent des espaces inconnus, favorisent leur valorisation et ont également leur part de responsabilité dans la gentrification, la mise en tourisme et la mutation immobilière d'espaces en déshérence pour le meilleur et pour le pire. On peut aussi craindre l'utilisation de l'artiste comme un pompier territorial, ou « couteau suisse de l'urbanisme », sommé d'intervenir rapidement, au dernier moment et sans beaucoup de moyens sur les situations les plus complexes.

Au-delà du phénomène d'" esthétisation du monde » que Walter Benjamin avait bien pressenti et que d'autres ${ }^{33}$ ont bien analysé, il faut prendre en compte les risques de folklorisation, de spectacularisation et de marchandisation ${ }^{34}$ des espaces et des temps de la métropole. Lorsqu'il n'y a plus que des événements singuliers porteurs chacun de la même valeur et de la même légitimité, alors tout devient effectivement sans signification : il n'y a plus ni différences ni mémoire mais seulement spectacle ${ }^{35}$. L'esthétisation du moindre interstice, du plus petit temps 
perdu (transport, chantier, etc.) interroge sur la notion de ville en continu sans friche, ni temps libre.

Dans un système à rotation rapide, il existe également un risque d'épuisement des lieux. Quand l'événement devient un spectacle trop régulier, l'émotion est congelée, le rite est folklorisé, la catharsis simulée, l'acteur devient spectateur et l'artiste prestataire. La multiplication des événements peut finir par saturer l'espace et les temps de la métropole, ne laissant plus assez de place à l'ennui, à la dérive, à l'improvisation et à l'errance qui restent des bonheurs urbains et des sources de sérendipité. Il y a, en effet, un risque pour que les nouvelles formes de l'art urbain, n'échappent pas à une approche du projet correspondant à une conception rationaliste et techniciste de l'action, « qui tend à écraser toute pensée de l'improvisation et du bricolage en refusant le jeu des circonstances ${ }^{36} »$.

Si les géo-artistes ne « changent pas le monde », ils changent déjà la ville et le regard que l'on peut porter sur elle. En ce sens, la rencontre géo-artistique ouvre à toute la richesse de la notion d'événement : « ouverture même d'une transformation ${ }^{37}$ ».

\section{Références}

Augoyard J.-F. (1979). Pas à pas. Essai sur le cheminement quotidien en milieu urbain. Paris, Seuil.

Bey H. (1997) TAZ. Zone autonome temporaire, Paris, L'Eclat.

Charlot A., Gwiazdzinski L. (2016), Géochorégraphie : marcher et danser avec Henri Maldiney, In Olivier Frérot, C. Younes, À l'épreuve de l'exister avec Henri Maldiney, Hermann, pp.313323.

Chaudoir P., «La Ville événementielle : temps de l'éphémère et espace festif », in Géocarrefour, Vol. 82/3.

Gwiazdzinski L. (2016), « Entre nouveaux imaginaires et mobilisations collectives. L'utopie du faire », in revue Urbia ${ }^{\circ} 16$, Lausanne, pp.123-144

Gwiazdzinski L. (dir.) (2016), L’hybridation des mondes, Grenoble, Elya. Hein F., Do It Yourself. Autodétermination et culture punk, Congé-sur-Orne, Le passager clandestin, 2012.

Lextrait F., Kahn F. (2005), Nouveaux territoires de l'art, Paris, Sujet-Objet. Novel A.S. (2013), La vie share. Mode d'emploi, Paris, Alternatives.

Paquot T. (Dir.) (2001), Le quotidien urbain, Paris, La découverte. Saez G.

Saez J.P. (2012), Les nouveaux enjeux des politiques culturelles. Dynamiques européennes, Paris, La découverte.

Sansot P. (2000), Chemins aux vents, Paris, Payot.

Sennet R. (2008), Ce que sait la main. La culture de l'artisanat, Paris, Albin Michel.

Soubeyran O. (2015), Pensée aménagiste et improvisation, Paris, Éditions des Archives Contemporaines.

\section{Notes}

1- Kracauer S., Rues de Berlin et d'ailleurs, Paris, Les Belles Lettres, 2013.

2- Michaud Y., L'Art à l'état gazeux : essai sur le triomphe de l'esthétique, Paris, Stock, 2004.

3- Bauman Z., Liquid Modernity, Cambridge, Polity Press, 2000. 
4- Ardenne P., Un Art contextuel : création artistique en milieu urbain, en situation, d'intervention, de participation, Paris, Flammarion, 2002.

5- Depardon R., Virilio P., Terre natale, Ailleurs commence ici, Paris, Fondation Cartier pour l'art contemporain, 2008.

6- Voir notamment les démarches de la Direction Générale de la Création Artistique avec la Mission nationale art et culture dans l'espace public ou le Plan-guide Arts et Aménagement des territoires.

7- Gwiazdzinski L., Chemins de traverse. La ville dans tous les sens, in Maud Le Floc'h, Mission repérage. Un élu un artiste, Éditions l'entretemps, 2006, pp. $2^{35}-244$.

8-Gwiazdzinski L., 2016, « La ville à l'épreuve des places », Tribune, Libération, 25 avril 2016.

9- Jambes J.P., Territoires apprenants. Esquisses pour le développement local du XXIe siècle,

Paris, L'Harmattan, 2001

10- Lévi-Strauss C., La Pensée sauvage, Paris, Plon, 1962.

11- Illich Y., Le Genre vernaculaire, Paris, Seuil, 1983.

12- Collectif ETC, Le Détour de France, une école buissonnière, Hypervilles, 2015. 13- De Certeau M., L'Invention du quotidien, Paris, Gallimard, 1988.

14- Voir notamment Lefebvre, 1961 ; De Certeau, 1988 ; Paquot, 2001 ; Sheringham, 2006. 15Caillois R., Les jeux et les hommes, Paris, Gallimard, 1967.

16- Debord G., Rapport sur la construction de situations, Paris, Mille et une nuits, 2000.

17- Morin E., «Éloge de la métamorphose », in Le Monde, 11 janvier, p. 18, 2010.

18- Goffman E., Les Rites d'interaction, Paris, Minuit, 1974.

19- Selon la belle expression de Maud Le Floc'h.

20- Rancière J., Le Partage du sensible, Paris, La Fabrique-éditions, 2000.

21- Dewey J., Art as Experience, New York, Penguin, 1980.

22- Lordon F., Imperium. Structures et affects des corps politiques, Paris, La Fabrique, 2015.

23- Maldiney H., Regard, Parole, espace, Lausanne, L'âge d'homme, 1994.

24- Maldiney H., « La rencontre et le lieu », in Younes C. (Dir.), Henry Maldiney. Philosophie, art et existence. La nuit surveillée, Paris, Cerf, 2007.

25- Lynch K., L'Image de la cité, Paris, Dunod, 1969.

26- Gwiazdzinski L., " La ville par intermittence : des temps de la fête à un urbanisme des temps ", in Revista Cidades, volume 8, n¹3, pp.317-335, 2011.

27- Claval P., La Logique des villes. Essai d'urbanologie, Paris, LITEC, 1981.

28- Faure A., « Les métropoles à l'épreuve de leur récit politique », Gouverner la métropole : pouvoirs et territoires. Bilan et directions de recherche. Metropolitan Representations, CEE LATTS APUR, Paris (France), 28, 29 \& 30 novembre 2012.

29- Matthey L., «Urbanisme fictionnel : l'action urbaine à l'heure de la société du spectacle », Métropolitiques, 28 octobre 2011.

30- Crozier M., Friedberg E., L'Acteur et le système, Paris, Seuil, 1977.

31- Gravari-Barbas M., « Iconicité et banalité », in Espaces urbains à l'aube du XXIe siècle, PUPS, pp. 87-108, 2010.

32- Boltanski L., Chiapello E., Le Nouvel esprit du capitalisme, Paris, Gallimard.

33- Lipovetsky G., Serroy J., L'Esthétisation du monde. Vivre à l'âge du capitalisme artiste, Paris, Gallimard, 2013.

34- Debord G., La société du spectacle, Paris, Buchet/Chastel, 1967.

35- Michaud Y., La Crise de l'art contemporain : utopie, démocratie et comédie, Paris, PUF, 1997.

36- Chevrier J.F., Des territoires, Paris, L'arachnéen, 2011. 
37- Maldiney H., Pulsion et présence, Psychanalyse à l’Université, tome 2, n5, décembre 1976.

(*) Luc Gwiazdzinski est géographe. Enseignant en aménagement et urbanisme à l'Université Joseph Fourier de Grenoble (IGA), il est responsable du Master Innovation et territoire et Président fondateur du Pôle des arts urbains. Chercheur au laboratoire Pacte (UMR 5194 CNRS) associé au MoTU (Université Biccoca et Politecnico de Milano) et à l'EREIST (Université Paris 1 Panthéon- Sorbonne), il oriente des enseignements et ses recherches sur les questions de mobilité, d'innovation métropolitaine et de chrono-urbanisme. Expert européen, il a dirigé de nombreux programmes de recherché, colloques internationaux, rapports, articles et ouvrages sur ces questions : L'hybridation des mondes, 2016, Elya ; Les ateliers de l'imaginaire, 2015, Elya ; Urbi et Orbi. Paris appartient à la ville et au monde, 2010, L'Aube ; Nuits d'Europe, 2007, UTBM ; Périphéries, 2007, L'harmattan ; La nuit dernière frontière de la ville, 2005, l'Aube ; Si la ville m'était contée, 2005, Eyrolles ; La nuit en questions (dir.), 2005, 1'Aube ; La ville 24 heures $/ 24$, 2003, L'Aube. Il a également dirigé une agence des temps et des mobilités, une agence de développement et une agence d'urbanisme et développement durable.

\section{Citer l'article :}

Gwiazdzinski L., 2016, Petite fabrique géo-artistique des espaces publics et des territoires, l'Observatoire nº 48 , été 2016, pp. 32-38

\section{Contact :}

lucmarcg@gmail.com 